\title{
Celiac Disease and Autoimmune Diseases
}

\author{
Mohsen Masjedi", Noushin Lotfi, Asma Eslami \\ Department of Immunology, Isfahan University of Medical Sciences, Isfahan, Iran
}

*Correspondence to Mohsen Masjedi;

Email: masjedi@med.mui.ac.ir

Received September 8, 2016 Revised September 20, 2016 Accepted September 28, 2016 Published online September 30, 2016

Please cite this article as follows: Masjedi M, Lotfi N, Eslami A. Celiac disease and autoimmune diseases. 2016;1(2):3536. doi:10.15171/ ijbms.2016.08.

\begin{abstract}
Celiac disease $(C D)$ is an inflammatory disorder of the small intestine. It consists of aberrant acquired immunity with presentation of CD toxic gluten peptides by HLA-DQ2 or HLA-DQ8 molecules to gluten-sensitive T cells. There is an increased prevalence of autoimmune diseases among celiac patients.
\end{abstract}

\section{Introduction}

Celiac disease (CD) leads to the infiltration of the epithelium with lymphocytes and tissue remodeling, resulting in villous atrophy. ${ }^{1,2}$

Some celiac patients do not have symptoms; because the healthy part of the intestine does not absorb enough nutrients to prevent symptoms' development. As just symptoms, some adults have fatigue and anemia. High incidences of cancers are seen in celiac patients. ${ }^{1,2}$

Other symptoms are amenorrhea, infertility, pyloric stenosis, ecchymosis and petechia (lack of vitamin K), edema, and dermatitis herpetiformis. Dermatitis herpetiformis is an autoimmune blistering disorder that is often associated with a gluten-sensitive enteropathy (GSE). ${ }^{1,2}$

Malabsorption of iron and vitamin B12 cause anemia. Malabsorption of calcium and vitamin D lead to osteoporosis, pathological fractures, bone and secondary hyperparathyroidism. ${ }^{1,2}$

$\mathrm{CD}$ has a range of symptoms. The most common symptoms include diarrhea, bloating, weight loss, and steatorrhea (fatty diarrhea). Other symptoms are fatigue, growth disorder in children, atrophy and muscle weakness. ${ }^{1,2}$

It is usually during infancy or early childhood (two weeks until one year old) that CD begins. It is possible that symptoms appear when children first begin to eat foods that contain gluten. In adults, symptoms may gradually emerge within a few months or even years. ${ }^{1}$
An increased prevalence of autoimmune disorders including autoimmune thyroid disease, type 1 diabetes mellitus, autoimmune liver diseases, and inflammatory bowel disease are detected in celiac patients. ${ }^{3}$

\section{Importance}

$\mathrm{CD}$, diabetes, and hypothyroidism appear together. In other words, many celiac patients are afflicted with hypothyroidism and diabetes. ${ }^{2,3}$

The remarkable point about the clinical symptoms of CD is that the pre-tests of the disease are not restricted to the gastrointestinal tract, but it includes a wide range from gastrointestinal or neurological asymptomatic or mild symptoms to severe malnutrition. ${ }^{2,3}$

\section{Diagnosis}

A multiplex assay combining insulin, glutamic acid decarboxylase (GAD), islet antigen-2 (IA-2) and transglutaminase autoantibodies is performed to facilitate the screening of the pre-type 1 diabetes and CD..$^{2,3}$

The enumeration of intraepithelial lymphocyte subsets (total, $\gamma \delta$, and CD3(-) IELs) by flow cytometry (FCM), named as IEL lymphogram, constitutes a useful tool for CD diagnosis. ${ }^{2,3}$

\section{Ethical Approval}

Not applicable.

\section{Competing Interests}

Authors declare that they have no competing interests.

Copyright (C) 2016 The Author(s); Published by Zabol University of Medical Sciences. This is an open-access article distributed under the terms of the Creative Commons Attribution License (http://creativecommons.org/licenses/by/4.0), which permits unrestricted use, distribution, and reproduction in any medium, provided the original work is properly cited. 


\section{References}

1. Wahab WA, Šuligoj T, Ellis J, et al. Coeliac disease: immunogenicity studies of barley hordein and rye secalinderived peptides. Int J Exp Pathol. 2016;97(4):303-309.

2. Abadie V, Discepolo V, Jabri B. Intraepithelial lymphocytes in celiac disease immunopathology. Semin Immunopathol. 2012;34(4):551-566.

3. Zhao Z, Miao D, Michels A, et al. A multiplex assay combining insulin, GAD, IA-2 and transglutaminase autoantibodies to facilitate screening for pre-type 1 diabetes and celiac disease. J Immunol Methods. 2016;430:28-32. 